Cipango Cahiers d'études japonaises

$21 \mid 2014$

Nouveaux regards sur les arts de la scène japonais II

\title{
Mesurer le temps au Japon
}

Le calendrier luni-solaire expliqué à l'aide de quelques notions astronomiques

Computing Time According to the Old Japanese Calendar

Jean Bazantay et Sylvie Yamazaki-Dubois

\section{(2) OpenEdition}

1 Journals

\section{Édition électronique}

URL : https://journals.openedition.org/cipango/2422

DOI : $10.4000 /$ cipango. 2422

ISSN : 2260-7706

Éditeur

INALCO

Édition imprimée

Date de publication : 31 décembre 2014

ISSN : 1164-5857

Référence électronique

Jean Bazantay et Sylvie Yamazaki-Dubois, « Mesurer le temps au Japon », Cipango [En ligne], 21 |

2014, mis en ligne le 29 novembre 2016, consulté le 30 juin 2021. URL : http://

journals.openedition.org/cipango/2422 ; DOI : https://doi.org/10.4000/cipango.2422

\section{(c) (i) (9)}

Cipango est mis à disposition selon les termes de la Licence Creative Commons Attribution - Pas d'Utilisation Commerciale 4.0 International. 


\section{Mesurer le temps au Japon Le calendrier luni-solaire expliqué à l'aide de quelques notions astronomiques}

\section{Computing Time According to the Old Japanese Calendar}

Jean BAZANTAY

CEJ, Inalco

Sylvie Yamazaki-Dubois

CLEA

Note des auteurs : Cet article est le fruit de recherches menées pour une activité interdisciplinaire associant mathématiques et japonais, que nous avons mise en place au Lycée FrancoJaponais de Tōkyō. Elle s'est déroulée sur plusieurs mois dans le cadre des IDD (Itinéraires de découverte) avec des classes de quatrième. Le compte rendu des activités proposées aux élèves a fait l'objet de publications dans le bulletin du Comité de Liaison Enseignants et Astronomes - CLEA : Yamazaki-Dubois Sylvie, Bazantay Jean, « Le calendrier luni-solaire japonais », Les Cahiers Clairaut, Nice, CLEA, 2014, n 146, p. 2-6; n 147, p. 30-35. 
« Le temps calendaire est le premier pont jeté par la pratique historienne entre le temps vécu et le temps cosmique » Paul Ricœur, 1983, Temps et récit, tome III, p. 154

\section{Introduction}

Le $12^{\mathrm{e}}$ mois de l'an 5 de Meiji fut d'une brièveté déconcertante pour le peuple japonais. Un rescrit impérial, publié à peine vingt jours auparavant, arrêta en effet

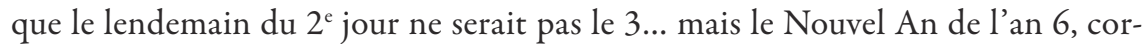
respondant au $1^{\mathrm{er}}$ janvier 1873 dans le calendrier grégorien (guregorio-reki グレゴ リ才暦). Cette décision qui fut préparée dans le plus grand secret correspond à l'adoption du calendrier grégorien ${ }^{1}$ connue en japonais sous le terme de kaireki 改 暦 (littéralement « réforme du calendrier »).

Jusqu'alors, pendant plus de mille deux cents ans, la computation du temps au Japon s'était effectuée suivant des calendriers luni-solaires (taiin taiyō reki 太陰太陽暦) d'inspiration chinoise et l'on peut imaginer l'onde de choc que cette annonce produisit dans tout le pays ${ }^{2}$. L'émoi fut d'autant plus grand que le calendrier était profondément lié à la vie quotidienne du peuple par toutes les superstitions et coutumes attachées aux dates ${ }^{3}$. Peu après le passage au calendrier grégorien, l'ancien système qui perdura quelque temps fut communément qualifié

1. Le système de décompte des heures fut aussi réformé. On passa d'un système de 12 périodes de durées variables selon les saisons (jüni shinkoku-sei 十二辰刻制) au système actuel des 24 heures fixes.

2. Dans les provinces éloignées, l'annonce du changement ne parvint que quelques jours à peine avant ce grand changement. Pour les détails concernant les préparatifs du nouveau calendrier et sa mise en œuvre, voir l'article de OKADA Yoshirō 岡田芳朗, «Kyûreki kara shinreki he, meiji kaireki no shinsō»旧暦から新暦へ 明治改暦の真相 (De l'ancien calendrier au nouveau calendrier, la vérité sur la réforme calendaire de Meiji), in KoYomi NO KAI (dir.), Koyomi no hyakka jiten 暦の百科事典 (Encyclopédie des calendriers), Tōkyō, Shinjinbutsuōrai-sha 新人物往来社, 1986, p. 153-158.

3. À cet égard, le dernier mois de l'année qui passa en quelque sorte « à la trappe » est un mois particulièrement important. 
d'《 ancien calendrier 》 (kyūreki 旧暦 $)^{4}$. Si cette dénomination fait aujourd'hui référence de manière générique à l'ancien système calendaire luni-solaire, elle désignait donc à l'origine plus spécifiquement le calendrier de Tenpō (Tenpō-reki 天 保歴), version la plus aboutie du calendrier luni-solaire en vigueur pendant les trente années qui précédèrent l'introduction du calendrier grégorien. Néanmoins, les forces navales japonaises ont perpétué la tradition en élaborant et en publiant le calendrier de Tenpō jusqu'en 2010. Ce faisant, elles poursuivaient un travail très ancien dévolu à la marine dans l'histoire des pays possédant des forces navales : la maîtrise du temps et des éphémérides qui était indispensable aux marins pour calculer la longitude de leur position.

Cet article a pour objectif de décrire le mode de computation des jours et des mois dans cet ancien calendrier luni-solaire japonais ${ }^{5}$. Il explique notamment l' intégration de la composante «solaire » avec le système des 24 souffles (nijüyon sekki二十四節気) permettant de fournir les repères climatiques indispensables à l'activité agricole et le fonctionnement du mois intercalaire (uruuzuki 閏月) pour éviter le « glissement » progressif des saisons dans le calendrier.

Pour illustrer nos explications et fournir une idée concrète des décalages avec le calendrier actuellement en vigueur, nous simulerons enfin le retour à un calendrier luni-solaire pour les années 2016 et 2017. Cela permettra aussi de comprendre la manière dont est fixée la date du Nouvel An « chinois » dans le calendrier grégorien.

Nous n'aborderons pas ici la dimension métaphysique et divinatoire des calendriers érigée en quasi-religion dans la voie du yin et du yang, ni les différentes croyances populaires et coutumes mentionnées dans les notes calendaires (rekich $\bar{u}$ 暦注). Ces aspects qui relèvent du rapport des Japonais au calendrier dépassent l'ambition de cet article, qui se limite à vouloir présenter les fondements astronomiques de l'ancien calendrier luni-solaire.

4. Francine Hérail précise que son usage officiel fut toléré jusqu'en 1910. Voir Francine Hérail, Histoire du Japon : des origines à la fin de Meiji, Paris, Publications Orientalistes de France, 1986, p. 22.

5. Nous prendrons comme référence le calendrier de Tenpō. 


\section{Introduction et développement des calendriers luni-solaires au Japon}

Avant l'introduction des premiers calendriers chinois dont les bases auraient été posées, selon Émile Biémont, dès le $3^{\mathrm{e}}$ millénaire avant Jésus-Christ, le déroulement du temps était rythmé par un calendrier naturel dont les subdivisions étaient fondées sur les périodes de travaux agraires ${ }^{6}$. L'introduction de la riziculture aux

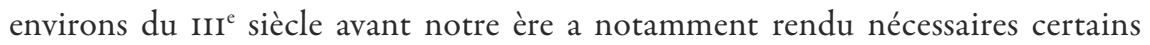
repères précis pour la préparation des rizières ou le repiquage dont rend compte le Gishi wajinden 魏志倭人伝 ${ }^{7}$. Comme l'explique Laurence Caillet, l'épanouissement de la fleur de kobushi (magnolia kobus) indique l'époque du labour de la rizière ; le début de la fonte des neiges sur une montagne donnée signale le début du printemps, etc ${ }^{8}$. L'alternance de saisons de travail et de repos, de périodes à caractère profane ou sacré rythme la vie quotidienne. Certaines fêtes et rites subsisteront d'ailleurs dans les nouveaux calendriers.

Le système chinois de computation du temps semble avoir été utilisé au Japon dès le $V^{e}$ siècle. Il a été introduit par l'intermédiaire d'astronomes du Royaume de Paekche, Kudara 百済 en japonais (région occidentale de l'actuelle Corée du Sud). Le livre 19 des Annales du Japon (Nihon shoki 日本書紀) consacré à l'Empereur Kinmei (509-571) fait mention de la contribution des astronomes de Paekche mandatés à cette fin. Toutefois, il semblerait que rapidement le Japon ne se soit plus satisfait de cette collaboration et qu'il ait cherché à contrôler lui-même l'élaboration de son calendrier. Pour cela, l'impératrice Suiko (554-628) fit venir en 602 un moine astronome, Kanroku 観勒, avec pour mission d'instruire des étrangers naturalisés. Cette initiative donna naissance au premier calendrier japonais, le calendrier de Genka (Genka-reki 元嘉暦) qui fut en vigueur pendant cinq ans à partir de l'an 652.

6. Emile BIÉmont, Rythmes du temps : astronomie et calendriers, Bruxelles, De Boeck, 2000, p. 274.

7. Source écrite la plus ancienne évoquant le Japon, datant du III $^{\mathrm{e}}$ siècle. Nom japonais d'un chapitre de la chronique officielle chinoise Sangoku shi 三国志 (Chroniques des trois royaumes) consacrée au royaume des Wei 魏 dans laquelle il est question des habitants du pays des Wo 倭.

8. Laurence Caillet, Fêtes et rites des quatre saisons au Japon, Cergy, Publications Orientalistes de France, 2002, p. 443. 
À partir du règne de l'empereur Tenmu (672), l'établissement des calendriers fut confié à des maîtres du yin et du yang (onmyōji 陰陽師) rattachés au 《 bureau du yin et du yang 》 (onmyōryō 陰陽寮) qui dépendait du ministère de la Maison impériale (nakatsukasa-shō 中務省). L'intégration de l'élaboration des calendriers à l'administration centrale montre l'importance qu'a très rapidement revêtue cette affaire. Le rôle des calendriers n'était plus la simple computation du temps ; ils devenaient des outils de divination de la voie du yin et du yang, religion astrophysique de l'antiquité chinoise que Bernard Franck décrit de la sorte :

Elle [la voie du yin et du yang] s'occupait de problèmes de calendrier, d'astronomie, d'astrologie, de géomancie, de divination, d'interprétation des présages. Reposant sur l'idée d'interrelations entre la Terre et le Ciel, entre la marche des choses humaines et celle du Cosmos, s'attachant à donner des conseils d'après les signes observés, elle était aussi nécessaire à l'État qu'aux particuliers9.

Dans les faits, le travail des spécialistes japonais était ainsi plus d'ordre astrologique et politique qu'astronomique. Francine Hérail rappelle que les maîtres du yin et du yang « se contentaient d'utiliser les traités chinois sans les modifier et que leur mission consistait surtout à déterminer le caractère faste ou néfaste des jours et des périodes et à interpréter les présages fournis par les phénomènes anormaux $\gg^{10}$. La dimension politique réside dans le souci de vouloir établir des chronologies nationales.

Le calendrier évolue ensuite à partir d'autres apports chinois adaptés au Japon. Du milieu du IX ${ }^{\mathrm{e}}$ siècle jusqu'à la fin du XVII ${ }^{\mathrm{e}}$ siècle, les calendriers japonais sont élaborés à partir du calendrier chinois de Senmyō (Senmyō-reki 宣明 暦 $)^{11}$ qui s'impose comme un modèle durable ${ }^{12}$. Cette pérennité va contribuer à la diffusion progressive d'informations relatives à l'élaboration des calendriers,

9. Bernard FrANK, Kata-imi et kata-tagae : étude sur les interdits de direction à l'époque Heian, Paris, Collège de France, 1998, p. 31.

10. Francine HÉraIL, op. cit., 1986, p. 21.

11. Plus que le calendrier lui-même, il s'agit en fait du mode de calcul (Senmyōreki-hō 宣 明暦法) ayant présidé à son élaboration.

12. En Chine, le calendrier de Senmyō ne resta en vigueur que 71 ans, de 822 à 892. 
qui étaient à l'origine secrètes. Avec l'affaiblissement du pouvoir de la Cour, la Maison impériale va perdre le monopole de l'établissement des calendriers et, à partir de l'époque de Kamakura, de nombreux calendriers régionaux non officiels (minkan-reki 民間暦) vont apparaittre dans tout le pays. Ces calendriers élaborés à partir de données chinoises présentent néanmoins quelques imprécisions que des astronomes japonais vont tenter de corriger à partir de l'époque d'Edo en tenant compte des coordonnées géographiques spécifiques du Japon. Le calendrier dit 《 de l'ère Jōkyō » (Jōkyō-reki 貞享暦) mis au point par Shibukawa Harumi 渋 川春海 et inauguré en l'an 2 de Jōkyōo (1685) marque une étape importante dans l'affranchissement vis-à-vis des calendriers chinois. Il s'agit en effet du premier calendrier officiel entièrement élaboré à partir de données et de calculs japonais.

Durant l'époque d'Edo, de nombreux traités scientifiques occidentaux, par exemple le traité d'astronomie de Lalande, ont également pénétré au Japon. Les informations collectées, concernant notamment la trajectoire elliptique de la Terre autour du Soleil et les variations induites de sa vitesse autour du Soleil décrites par la $2^{\mathrm{e}}$ loi de $\operatorname{Kepler}^{13}$, ont permis d'améliorer par phases successives le calendrier de Jōkyō pour aboutir au calendrier de Tenpō, dernier calendrier luni-solaire en vigueur.

\section{Principe du calendrier luni-solaire}

L'observation du mouvement des astres est à la base de tous les calendriers dont les références sont des cycles naturels réguliers. Le premier de ces cycles, l'alternance du jour et de la nuit, conduit à une première unité : le jour, défini par la rotation de la Terre sur son axe.

Deux autres rythmes célestes se sont aussi imposés : la révolution de la Lune autour de la Terre qui s'observe depuis cette dernière par la succession des phases et la révolution de la Terre autour du Soleil qui se traduit par le retour des saisons. Mais ces deux cycles n'entretiennent pas de rapport mathématique simple entre eux et leur harmonisation est difficile. Un calendrier lunaire est utile aux pêcheurs pour connaître les marées (dont le lien avec les phases de la Lune a été très tôt

13. « Le segment de droite qui relie le centre du Soleil au centre de la Terre balaie des aires égales pendant des temps égaux. » 
constaté) tandis que les agriculteurs tirent profit d'un calendrier solaire pour juger des périodes les plus favorables aux différents travaux. Le calendrier luni-solaire essaie de concilier ces deux exigences.

\section{Partie lunaire}

Aisément identifiables à l'œil nu, les différentes phases de la Lune constituent un cycle de 29,53 jours appelé « lunaison » correspondant à la durée de la révolution de la Lune autour de la Terre. Dans un calendrier lunaire, les mois sont calés sur ces lunaisons. 29,53 n'étant pas un nombre entier, dans la pratique l'alternance de mois de 29 et de 30 jours s'est naturellement imposée. Ces mois sont respectivement appelés « petit mois » (shō no tsuki 小の月) et « grand mois » (dai no tsuki 大の月). Cette alternance n'est toutefois pas régulière et il pouvait arriver que plusieurs mois ayant le même nombre de jours se suivent pour corriger l'approximation (29,53 étant plus proche de 30 que de 29, les mois de 30 jours seront plus nombreux que les mois de 29 jours).

Le premier calendrier de Tenpō datant de l'an 6 de l'ère Kaei (1853) comptait par exemple 355 jours répartis en mois de la manière suivante :

\section{DOCUMENT 1.}

REPRODUCTION D'UNE PARTIE DE LA PREMIÈRE PAGE DU CALENDRIER DE L'AN 6

\begin{tabular}{|c|c|c|c|}
\hline \multicolumn{4}{|c|}{ DE KAEI } \\
\hline 十 & 七 & 四 & 正 \\
\hline 月 & 月 & 月 & 月 \\
\hline 大 & 小 & 大 & 大 \\
\hline 十 & 八 & 五 & 二 \\
\hline 二 & 月 & 月 & 月 \\
\hline $\begin{array}{l}\text { 月 } \\
\text { 小 }\end{array}$ & 大 & 小 & 小 \\
\hline 十 & 九 & 六 & 三 \\
\hline 二 & 月 & 月 & 月 \\
\hline 月 & 小 & 大 & 大 \\
\hline 大 & & & \\
\hline
\end{tabular}

On remarque que les $3^{e}$ et $4^{e}$ mois étaient des « grands mois ». Par convention, les mois lunaires commencent toujours à la nouvelle lune qui correspond au moment où la Lune est invisible depuis la Terre. 


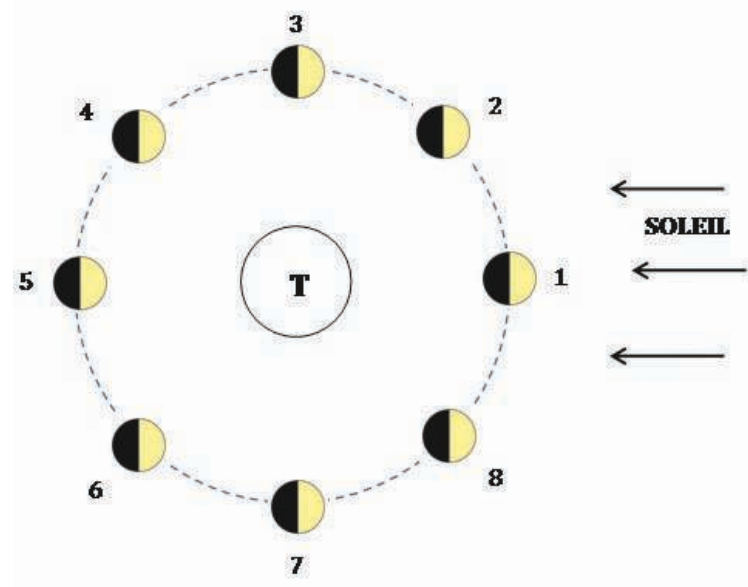

\begin{tabular}{|l|l|l|l|l|l|l|l|}
\hline 1 & 2 & 3 & 4 & 5 & 6 & 7 & 8 \\
\hline & & & & & & & \\
\hline
\end{tabular}

Le premier schéma représente les phases de la lune vues par un observateur extérieur au système Terre-Lune. Le tableau ci-après montre ces mêmes phases vues de la Terre.

Remarque : sur le schéma, la position 5 laisserait penser que, la Terre arrêtant les rayons du Soleil, la Lune se trouve dans l'ombre et qu'il se produit alors une éclipse de Lune. De même on pourrait penser que, dans la position 1, la Lune cache le Soleil aux observateurs terrestres ce qui entraîne une éclipse de Soleil. En réalité, le plan orbital de la Lune autour de la Terre est légèrement incliné par rapport à celui de la Terre autour du Soleil et l'alignement Terre-Lune-Soleil ne se produit pas à chaque lunaison.

Le prochain tableau présente le décompte des jours dans chaque mois lunaire. 
DOCUMENT 3.

LES JOURS D'UN MOIS LUNAIRE

\begin{tabular}{|c|c|c|c|}
\hline Jour & Phase & Nom de la phase & Autre nom/remarques \\
\hline jer Jour & & $\begin{array}{l}\text { 新月 shingetsu* } \\
\text { Nouvelle lune }\end{array}$ & $\begin{array}{l}\text { 朔 saku (moment précis du passage de la Lune } \\
\text { entre la Terre et le Soleil) }\end{array}$ \\
\hline 2e Jour & & $\begin{array}{l}\text { 二日月 futsukazuki } \\
\text { Lune du } 2^{\mathrm{e}} \text { jour }\end{array}$ & $\begin{array}{l}\text { Comme la nouvelle lune, la lune du } 2^{\mathrm{e}} \text { jour est } \\
\text { quasiment invisible à l'œil nu. }\end{array}$ \\
\hline 3e Jour & & $\begin{array}{l}\text { 三日月 mikazuki } \\
1^{e r} \text { croissant }\end{array}$ & $\begin{array}{l}\text { Autre graphie: 朏 } \\
\text { 眉月 mayuzuki, 若月 wakazuki, 初月 shogetsu, etc. }\end{array}$ \\
\hline 4e Jour & & $\begin{array}{l}\text { 四日月 yokkazuki } \\
\text { Lune du } 4^{e} \text { jour }\end{array}$ & \\
\hline 5 e Jour & & $\begin{array}{l}\text { 五日月 itsukazuki } \\
\text { Lune du } 5^{e} \text { jour }\end{array}$ & \\
\hline 6e Jour & & $\begin{array}{l}\text { 六日月 muikazuki } \\
\text { Lune du } 6 \text { e jour }\end{array}$ & \\
\hline 7e Jour & & $\begin{array}{l}\text { 七日月 nanokazuki } \\
1^{\mathrm{er}} \text { quartier }\end{array}$ & $\begin{array}{l}\text { 上弦の月 jógen no tsuki (littéralement « lune du } 1^{\text {er }} \\
\text { arc } \text {, nom donné en raison de l'aspect de la lune au } \\
\text { moment où elle se couche) }\end{array}$ \\
\hline $8^{e}$ Jour & & $\begin{array}{l}\text { 八日月 yōkazuki } \\
1^{\text {er quartier }}\end{array}$ & $\begin{array}{l}\text { Cette lune se lève aux alentours de midi et se } \\
\text { couche au milieu de la nuit. }\end{array}$ \\
\hline ge Jour & & $\begin{array}{l}\text { 九日月 kokonokazuki } \\
\text { Lune du ge jour }\end{array}$ & \\
\hline $10^{e}$ Jour & & $\begin{array}{l}\text { 十日月 tôkazuki } \\
\text { Lune du } 10^{e} \text { jour }\end{array}$ & \\
\hline
\end{tabular}

- C'est sous linfluence de l'astronomie occidentale que l'on a nommé « nouvelle lune » cette phase où la lune est paradoxalement invisible à l'ceil nu. Autrefois la nouvelle lune désignait le premier croissant de lune très fin visible au crépuscule dans le ciel dioccident après la conjonction mensuelle. Dans le calendrier gaulois, également luni-solaire, les mois lunaires commençaient au $6^{\mathrm{e}}$ jour de la lunaison, lorsqu'une partie du disque est dejaja éclairée. 


\section{CIPANGO}

394 Cahiers d'études japonaises n²1

\begin{tabular}{|c|c|c|}
\hline 11 Jour & $\begin{array}{l}\text { 十一日月 } \\
\text { jüchinichizuki } \\
\text { Lune du } 11^{e} \text { jour }\end{array}$ & \\
\hline $12^{\mathrm{e}}$ Jour & $\begin{array}{l}\text { †二日月 } \\
\text { jûninichizuki } \\
\text { Lune du } 12^{e} \text { jour }\end{array}$ & \\
\hline 13e Jour & $\begin{array}{l}\text { †三日月 } \\
\text { jusannichizuki } \\
\text { Lune du } 13^{e} \text { jour }\end{array}$ & $\begin{array}{l}\text { 十三夜 jüsan.ya }\left(\alpha 13^{e} \text { nuit »). Ce terme fait parfois }\right. \\
\text { référence à la lune du } 13^{e} \text { jour du } 9^{e} \text { mois*. }\end{array}$ \\
\hline 14 e Jour & $\begin{array}{l}\text { 十四日月 } \\
\text { jüyokkazuki } \\
\text { Lune du } 14^{e} \text { jour }\end{array}$ & 小望月 komochizuki \\
\hline 15 e Jour & $\begin{array}{l}\text { 十五日月 } \\
\text { jügonichizuki } \\
\text { Pleine lune }\end{array}$ & $\begin{array}{l}\text { 十五夜 jügoya ( } 15^{e} \text { nuit). En particulier la lune du } \\
15^{\mathrm{e}} \text { jour du } 8 \mathrm{e} \text { mois connue sous le nom de } \text { a belle } \\
\text { lune du milieu de l'automne } \text { (中秋の名月 chüshù } \\
\text { no meigetsu) qui faisait l'objet d'une contemplation } \\
\text { toute particulière**. } \\
\text { 満月 mangetsu, 望月 mochizuki } \\
\text { Apparait à la tombée du jour. }\end{array}$ \\
\hline 16 e Jour & $\begin{array}{l}\text { 十六日月 } \\
\text { jürokunichizuki } \\
\text { Lune du } 16 \text { e jour }\end{array}$ & $\begin{array}{l}\text { 十六夜 izayoi (nom dérivé du verbe izayou, } \alpha \text { hési- } \\
\text { ter », en référence au lever de lune qui se produit } \\
\text { presque une heure après celui de la veille***. } \\
\text { 有明月 ariakezuki, lune de l'aube****. }\end{array}$ \\
\hline 17 e Jour & $\begin{array}{l}\quad \text { 十七日月 } \\
\text { jüshichinichizuki } \\
\text { Lune du } 17 \text { jour }\end{array}$ & $\begin{array}{l}\text { 立待月 tachimachizuki (littéralement } * \text { lune que } \\
\text { l'on attend debout } * \text { ), allusion au lever de lune qui } \\
\text { n'est pas encore trop tardif. }\end{array}$ \\
\hline 18 Jour & $\begin{array}{l}\text { 十八日月 } \\
\text { jühachinichizuki } \\
\text { Lune du } 18^{e} \text { jour }\end{array}$ & $\begin{array}{l}\text { 居待月 imachizuki ( } \propto \text { lune que l'on attend assis } ») \text { : il } \\
\text { devient trop fatigant de rester debout pour at- } \\
\text { tendre le lever de lune! }\end{array}$ \\
\hline 19 e Jour & $\begin{array}{l}\text { 十九日月 } \\
\text { jūkunichizuki } \\
\text { Lune du 19e jour }\end{array}$ & $\begin{array}{l}\text { 寝待月 nemachizuki (« lune quón attend couché *) } \\
\text { 卧待月fushimachizuki (idem) }\end{array}$ \\
\hline $20^{\circ}$ Jour & $\begin{array}{l}\begin{array}{l}\text { 二十日月 } \\
\text { hatsukazuki } \\
\text { Lune du } 20^{e} \text { jour }\end{array} \\
\text { Lun }\end{array}$ & 更待月 fukemachizuki ( lune qu'on attend couché ») \\
\hline
\end{tabular}

- Celle-cia pour autre nom * lune des pois s (mame meigetsu 豆名月).

$*$ Le $8^{e}$ mois correspondait au milieu de l'automne et, en pleine saison des typhons, quand la pleine lune apparaissait dans un ciel balayé et purifié, on en ressentait toute la beauté et la fraicheur. Les offrandes étaient principalement composées de patates douces fraíchement récoltées, d'où un autre nom de cette lune : e la lune des tubercules s (imo meigetsu 芋名月).

$*$ La lune se lève en moyenne 50 minutes plus tard chaque jour. II y a toutefois dimportantes variations selon les latitudes (plus on est au nord, plus la durée entre deux levers de lune est importante) et la période de l'année (les amplitudes sont les plus faibles au moment de l'équinoxe d'automne et les plus fortes au moment de léquinoxe de printemps).

$\cdots$ Nom genérique donné a toutes les lunes à partir du $16^{\mathrm{e}}$ jour en raison du fait quélles sont encore visibles après l'aube. 


\begin{tabular}{|c|c|c|}
\hline $21^{e}$ Jour & $\begin{array}{c}\text { ニ十一日月 } \\
\text { nijüichinichizuki } \\
\text { Lune du } 21^{\mathrm{e}} \text { jour }\end{array}$ & \\
\hline $22^{\mathrm{e}}$ Jour & $\begin{array}{l}\text { 二十二日月 } \\
\text { nijüninichizuki } \\
\text { 2e quartier }^{\text {e }}\end{array}$ & \multirow{2}{*}{ 下弦の月 kagen no tsuki (lune du $2^{e}$ arc) } \\
\hline $23^{2}$ Jour & $\begin{array}{c}\text { 二十三日月 } \\
\text { nijüsannichizuki } \\
2^{e} \text { quartier }\end{array}$ & \\
\hline 24 e Jour & $\begin{array}{c}\text { 二十四日月 } \\
\text { nijüyokkazuki } \\
\text { Lune du } 24^{e} \text { jour }\end{array}$ & \\
\hline $25 \mathrm{e}$ Jour & $\begin{array}{c}\text { 二十五日月 } \\
\text { nijügonichizuki } \\
\text { Lune du } 25^{e} \text { jour }\end{array}$ & \\
\hline $26^{\mathrm{e}}$ Jour & $\begin{array}{c}\text { 二十六日月 } \\
\text { nijürokunichizuki } \\
\text { Lune du } 26 \mathrm{e} \text { jour }\end{array}$ & \\
\hline $27^{e}$ Jour & $\begin{array}{l}\text { 二十七日月 } \\
\text { nijüshichinichizuki } \\
\text { Lune du } 27^{\mathrm{e}} \text { jour }\end{array}$ & \\
\hline $28^{e}$ Jour & $\begin{array}{l}\text { ニ十八日月 } \\
\text { nijūhachinichizuki } \\
\text { Lune du } 28^{e} \text { jour }\end{array}$ & \\
\hline 29 e Jour & $\begin{array}{c}\text { 二十九日月 } \\
\text { nijūkunichizuki } \\
\text { Lune du } 29^{\mathrm{e}} \text { jour }\end{array}$ & \multirow{2}{*}{$\begin{array}{l}\text { 晦 tsugomori (autre transcription : 月爝り) } \\
\text { Lune invisible à l'œeil nu. }\end{array}$} \\
\hline $30^{e}$ Jour & $\begin{array}{c}\text { 三十日月 misokazuki } \\
\text { Lune du } 30^{e} \text { jour }\end{array}$ & \\
\hline
\end{tabular}

Les mois étant définis, une unité supérieure est nécessaire pour le décompte des périodes plus longues. Le rythme des saisons offre une unité naturelle profondément ancrée dans la vie humaine et, pour que le retour des saisons se fasse à date fixe, il faut tenir compte de l'année solaire. Dès l'époque de la dynastie Han (de 206 av. J.-C. à 220 ap. J.-C.), les astronomes chinois considéraient que sa 
durée était de 365,25 jours $^{14}$. Elle comporte un peu plus de 12 lunaisons $(365,25$ jours $/ 29,53=12,37$ ) qui vont servir de base à la définition de l'année calendaire.

Si l'année calendaire ne comportait que 12 mois, elle serait alors d'une durée moyenne de 354,36 jours $(29,53 \times 12)$ et aurait donc environ 11 jours de moins que l'année solaire. En conséquence, les saisons «glisseraient » d'autant chaque année. Pour corriger cette dérive, le calendrier luni-solaire introduit périodiquement des années de 13 mois appelées années embolismiques.

On peut remarquer que 19 années solaires coïncident presque exactement avec 235 lunaisons $(365,25 \times 19=6939,75$ et $29,53 \times 235=6939,55) .235$ lunaisons correspondent à 19 années de 12 mois lunaires auxquelles il faut rajouter 7 mois $(235=19 \times 12+7)$. Il faut donc introduire 7 années embolismiques en 19 ans pour mettre en phase le calendrier lunaire avec les saisons. Ce cycle de 19 ans est connu sous le nom de cycle de Méton d'après l'astronome grec qui l'aurait établi au $\mathrm{V}^{\mathrm{e}}$ siècle av. J.-C. Bernard Frank signale que certaines sources indiquent que les Chinois l'auraient également découvert vers le $\mathrm{VI}^{\mathrm{e}}$ siècle av. J.-C. ${ }^{15}$.

\section{Partie solaire : les 24 souffles}

Dès le règne de la dynastie Yin (1766-1122 av. J.-C.), des calendriers luni-solaires sont attestés en Chine. Pour fournir des repères fixes indiquant le rythme des saisons, un système de 24 souffles désignant 24 repères temporels a été mis au point.

14. L'année solaire peut se définir et se mesurer de plusieurs façons. Vue de l'espace, la Terre tourne autour du Soleil, mais, pour un observateur terrestre, c'est le Soleil qui se déplace sur le fond du ciel dans une zone appelée écliptique sur laquelle se trouvent les constellations du zodiaque. On peut donc mesurer le temps que met le Soleil à retrouver la même position par rapport aux étoiles de la sphère céleste. Il s'agit alors de l'année sidérale qui est actuellement évaluée à 365,256 jours. On peut aussi déterminer les instants des solstices ou des équinoxes et mesurer l'intervalle séparant deux événements identiques. La durée entre deux équinoxes de printemps (dans l'hémisphère nord) est appelée année vernale (anciennement année tropique) et est actuellement évaluée à 365,242 jours. Cette durée est plus courte que l'année sidérale à cause d'un phénomène appelé précession des équinoxes qui fait que la ligne des équinoxes n'est pas fixe dans l'espace.

15. Bernard Frank, Démons et jardins : aspects de la civilisation du Japon ancien, Paris, Collège de France - Institut des Hautes Études Japonaises, 2011. 
Suivant les sources, l'emploi du terme sekki 節気 est sujet à variations. Il désigne tantôt de manière générique ces 24 points, tantôt seulement douze d'entre eux, correspondant à des points d'articulation saisonniers (setsu 節, nœud, jointure, articulation). Les douze autres sont qualifiés de $c h \bar{u}$ 中 ou $c h \bar{u} k i$ 中気. Nous adopterons ici la distinction opérée par Bernard Frank, qui utilise le terme de $k i$ 気 (souffle) pour l'ensemble et réserve celui de sekki aux douze points d'articulation qu'il nomme « souffles initiaux ». Le terme chüki est traduit par « souffle médian ${ }^{16}$.

Dans l'ancien calendrier, les souffles ont été d'abord calculés à partir du solstice d'hiver en divisant la durée de l'année solaire par 24. La durée séparant deux souffles était donc de 15,22 jours (365,25/24).

Cette méthode de calcul, connue sous le nom de « méthode régulière » (heikihō 平気法), ne tient pas compte des variations de la vitesse de révolution de la Terre autour du Soleil (liées à l'ellipticité de son orbite) et a montré ses limites dans la prévision très approximative de certaines dates d'éclipses.

Ces variations s'expriment par la deuxième des trois lois appelées « Lois de Kepler », publiée en 1609 et qui s'énonce ainsi : « Le segment de droite qui relie le centre du Soleil au centre de la Terre balaie des aires égales pendant des temps égaux. »

DOCUMENT 4.

ORBITE DE LA TERRE AUTOUR DU SOLEIL

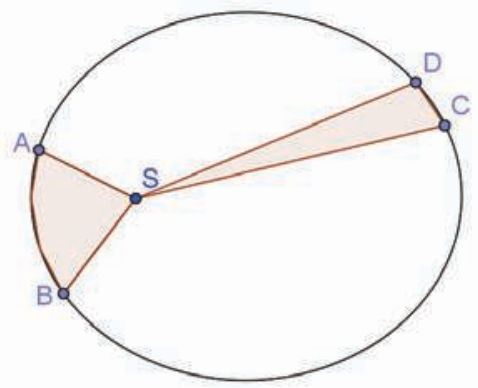

Sur ce schéma, les aires grisées sont égales; la Terre met le même temps pour parcourir les arcs $\mathrm{AB}$ et $\mathrm{CD}$.

16. Bernard Frank, op. cit., 1998. 
Une conséquence de ces variations de vitesse est que d'un souffle à l'autre les distances parcourues par la Terre sont inégales, et il en résulte une certaine imprécision des repères saisonniers.

Toutefois, en l'absence de méthode plus précise de mesure du mouvement des astres, la « méthode régulière » a longtemps été la seule disponible. À partir du calendrier connu sous le nom de calendrier de Jiken (Jiken-reki 時憲暦) en vigueur durant la dynastie Qing (1645-1911), la méthode de détermination des souffles s'est affinée en se basant cette fois sur la distance parcourue par la Terre pendant une année. L'orbite de la Terre (360 degrés) a été divisée en 24, ce qui conduit à des angles de 15 degrés entre deux souffles consécutifs. Pour un observateur terrestre, cela revient à mesurer des déplacements de 15 degrés de la position du Soleil sur l'écliptique. C'est la durée entre deux souffles consécutifs qui devient donc variable, la variation allant de 14,72 à 15,72 jours. Cette méthode est connue sous le nom de «méthode définie » (teiki-hō 定気法). Le calendrier japonais de Tenpō fonctionnait suivant ce système. En indiquant ces points sur le calendrier lunaire, on a pu intégrer plus précisément le rythme des saisons et l'année solaire.

Les souffles initiaux indiquent les débuts de changements climatiques alors que les souffles médians correspondent à l'apogée et au basculement vers le climat suivant. Les intervalles entre deux souffles initiaux ou médians sont de 30,44 jours $(15,22 \times 2)$ pour la première méthode et varient de 29,44 à 31,44 jours pour la deuxième. Chacun de ces intervalles étant proche de la durée d'une lunaison (29,5 jours), la plupart des mois lunaires contiennent donc un souffle initial et un souffle médian. Le $2^{\mathrm{e}}$ mois ${ }^{17}$ inclut par exemple le réveil des insectes (keichitsu 啓 蟄) et l'équinoxe de printemps (shunbun 春分). Toutefois, la plupart de ces intervalles étant légèrement supérieurs à 29,5 , certains mois lunaires ne comporteront qu'un seul des deux souffles. Pour un type de souffle donné (initial ou médian), ce phénomène se répétera tous les deux ou trois ans.

17. Exemples tirés du calendrier de l'an 6 de Kaei (1853) correspondant au premier calendrier Tenpō. 
DOCUMENT 5.

LES 24 SOUFFLES

\begin{tabular}{|c|c|c|c|c|}
\hline $\begin{array}{l}\text { Souffle } \\
\text { initial }\end{array}$ & $\begin{array}{l}\text { Souffle } \\
\text { médian }\end{array}$ & $\begin{array}{l}\text { Transcription } \\
\text { chinoise }\end{array}$ & rōmaji & Traduction $^{*}$ \\
\hline \multirow[t]{2}{*}{1} & & 立春 & risshun & Commencement du printemps \\
\hline & 1 & 雨水 & usui & Eau de pluie (pluies) \\
\hline \multirow[t]{2}{*}{2} & & 啓蟄 & keichitsu & Réveil des insectes \\
\hline & 2 & 春分 & shunbun & Équinoxe de printemps \\
\hline \multirow[t]{2}{*}{3} & & 清明 & seimei & Lumière pure \\
\hline & 3 & 穀雨 & kokuu & $\begin{array}{l}\text { Pluies des céréales (pluie de } \\
\text { grains) }\end{array}$ \\
\hline \multirow[t]{2}{*}{4} & & 立夏 & rikka & Commencement de l'été \\
\hline & 4 & 小満 & shōman & $\begin{array}{l}\text { Les épis se forment (petite abon- } \\
\text { dance) }\end{array}$ \\
\hline \multirow[t]{2}{*}{5} & & 芒種 & bōshu & Les céréales ont de la barbe \\
\hline & 5 & 夏至 & geshi & Solstice d'été \\
\hline \multirow[t]{2}{*}{6} & & 小暑 & shōsho & $\begin{array}{l}\text { Chaleur modérée (petites cha- } \\
\text { leurs) }\end{array}$ \\
\hline & 6 & 大暑 & taisho & Grande chaleur (grandes chaleurs) \\
\hline \multirow[t]{2}{*}{7} & & 立秋 & risshū & Commencement de l'automne \\
\hline & 7 & 處暑 & shosho & Fin de la chaleur (fin des chaleurs) \\
\hline \multirow[t]{2}{*}{8} & & 白露 & hakuro & Rosée blanche \\
\hline & 8 & 秋分 & shūbun & $\begin{array}{l}\text { Équinoxe } \\
\text { d’automne }\end{array}$ \\
\hline \multirow[t]{2}{*}{9} & & 寒露 & kanro & Rosée froide \\
\hline & 9 & 霜降 & sōkō & Gelée blanche (arrivée du givre) \\
\hline \multirow[t]{2}{*}{10} & & 立冬 & rittō & Commencement de l'hiver \\
\hline & 10 & 小雪 & shōsetsu & $\begin{array}{l}\text { Neige peu abondante (petite } \\
\text { neige) }\end{array}$ \\
\hline \multirow[t]{2}{*}{11} & & 大雪 & taisetsu & Neige abondante \\
\hline & 11 & 冬至 & tōji & Solstice d'hiver \\
\hline \multirow[t]{2}{*}{12} & & 小寒 & shōkan & Froid peu intense \\
\hline & 12 & 大寒 & daikan & Grand froid (grands froids) \\
\hline
\end{tabular}

" Traductions reprises de FRANK Bernard, Kata-imi et kata-tagae : Étude sur les interdits de direction à l'époque Heian, Paris, Collège de France, 1998. Les traductions indiquées entre parenthèses sont celles de CAILLET Laurence, Fêtes et rites des quatre saisons au Japon, Cergy, Publications Orientalistes de France, 2002. 
Parmi ceux-ci, les équinoxes, les solstices ainsi que les quatre débuts de saisons désignés sous le terme de hassetsu 八節 (《 huit souffles importants 》) revêtent une importance particulière.

Pour rendre compte plus finement des variations saisonnières, les astronomes chinois ont divisé chaque souffle en trois périodes ou climats ( $k \bar{o}$ 候) d'une durée d'environ cinq jours chacun. L'année comptait ainsi 72 climats (shichijū ni kō 七 十二候). Le nom de chaque climat exprimé sous la forme d'une courte phrase rendait compte d'un phénomène naturel spécifique de la période. Avec le système des souffles, les Japonais ont adopté cette subdivision en l'adaptant partiellement aux spécificités naturelles et culturelles japonaises. Longtemps, les Japonais ont donc eu comme références climatiques des codes chinois. Ce n'est qu'à l'époque d'Edo qu'un groupe d'astronomes dirigé par Shibukawa Harumi 渋川春海 a mis au point une terminologie adaptée aux conditions naturelles japonaises, connue sous le nom des « 72 climats de notre Cour » (honchō shichi jü ni kōo 本 朝七十二候). Ces 72 climats qui figurent encore aujourd'hui sur certains calendriers n'ont pas évolué depuis le premier calendrier grégorien. Les Japonais y font encore référence pour des observations naturelles et dans certaines salutations saisonnières codifiées appartenant à la correspondance.

\section{Le mois intercalaire}

Comme nous l'avons expliqué dans la première partie, pour éviter la dérive progressive des saisons dans le calendrier lunaire tout en conservant des mois de 29 ou 30 jours, il faut rajouter un mois tous les deux ou trois ans.

Par ailleurs c'est, comme nous venons de le voir, à cette même périodicité qu'on rencontre un mois lunaire ne comportant pas de souffle médian. Par convention, il a été décidé que ce mois particulier serait le mois supplémentaire nommé uruuzuki ${ }^{18}$ nécessaire au rattrapage des saisons. On lui attribue le même numéro que le précédent, pour préserver la correspondance des mois avec les repères saisonniers ${ }^{19}$.

18. Littéralement « mois surnuméraire ». La dénomination courante de « mois intercalaire » ne correspond donc pas à la traduction littérale mais plutôt à la fonction de ce mois.

19. Un autre avantage de cette numérotation était peut-être de mieux faire passer la baisse de pouvoir d'achat des fonctionnaires payés à l'année. 
MESURER LE TEMPS AU JAPON

\section{À propos des saisons}

Les saisons peuvent être appréhendées de différentes façons. Dans les zones tempérées, elles correspondent à des climats spécifiques : l'hiver est la période la plus froide, l'été la plus chaude et le printemps et l'automne sont des périodes intermédiaires ; les dates de changement de saison correspondent plus ou moins à des points charnières. Dans la culture, les saisons sont aussi associées au cycle naturel du vivant : (re)naissance, croissance, apogée, déclin, mort.

En Occident, le début des saisons est indiqué par les équinoxes et les solstices. L'été s'étend par exemple du solstice d'été ( 21 ou 22 juin) à l'équinoxe d'automne (21 ou 22 septembre). Aux latitudes moyennes de l'hémisphère nord, cela correspond au moment de l'année où il fait le plus chaud.

Au Japon, les quatre ritsu 立 ( «lever »), risshun, rikka, risshü et rittō, indiquent le début des saisons calendaires. D'un point de vue climatique, ces dates ne correspondent pas à l'idée que nous nous faisons du climat de chaque saison. Il est ainsi fréquent qu'il fasse très froid après le risshun qui indique le début du printemps ou inversement très chaud passé le risshù signalant le début de l'automne. Passées ces dates, on parlera alors respectivement de yokan 余寒 (《froid subsidiaire 》) ou de zansho 残暑 (《chaleur persistante ») comme s'il s'agissait d'anomalies. Cette perception des choses est d'ailleurs attestée dans des salutations saisonnières stéréotypées telles que «Bien que nous soyons au printemps... »(暦の上春で すが...) dans lesquelles on signale le grand décalage entre la réalité climatique et la saison calendaire.

Cette précocité des saisons est parfois attribuée à un héritage du calendrier luni-solaire. Il n'en est rien car ces souffles sont liés à la partie solaire du calendrier. Cela renvoie en fait à la définition du concept de saison dans la tradition chinoise. Littéralement, risshun peut se traduire « le printemps se dresse » ou «le printemps prend son départ ${ }^{20}$. Cela ne signifie pas que le printemps soit

20. Frank rappelle qu'en Chine l'établissement des saisons « $l i »$ (立) était marqué par un rite d'accueil accompli par le monarque qui allait « au-devant de chaque saison ». Les Japonais ne semblent pas avoir attribué au mot ritsu un sens se rapportant à des notions aussi précises. Voir Bernard FrANK, op. cit., 2011, p. 303-304. 
arrivé mais plutôt qu'à partir de cette date la douceur va commencer à arriver. En d'autres termes, cela indique le pic du froid. Entendu dans ce sens, il ne paraît plus surprenant qu'il fasse froid au moment du risshun. Ce serait même plutôt la norme. Cette méthode de détermination des saisons est connue sous le nom de 《 découpage par setsu» (setsugiri 節切り).

Cependant, comme l'attestent les salutations signalant un décalage entre le temps qu'il fait et la saison calendaire, bon nombre de Japonais semblent avoir oublié cette définition originelle des saisons et avoir intégré la perception occidentale.

En concurrence avec cette méthode, une définition des saisons selon un découpage mensuel est également attestée. Suivant celle-ci, les trois premiers mois de l'année correspondent au printemps, les trois suivants à l'été et ainsi de suite. Cette méthode est baptisée « découpage par mois » (tsukigiri 月切り). Selon cette seconde méthode, le début de l'année coïncide avec le début du printemps calendérique. Dans la pratique quotidienne, ces deux méthodes semblaient ne pas être exclusives l'une de l'autre mais se superposer.

DOCUMENT 6.

LES SAISONS CALENDAIRES JAPONAISES

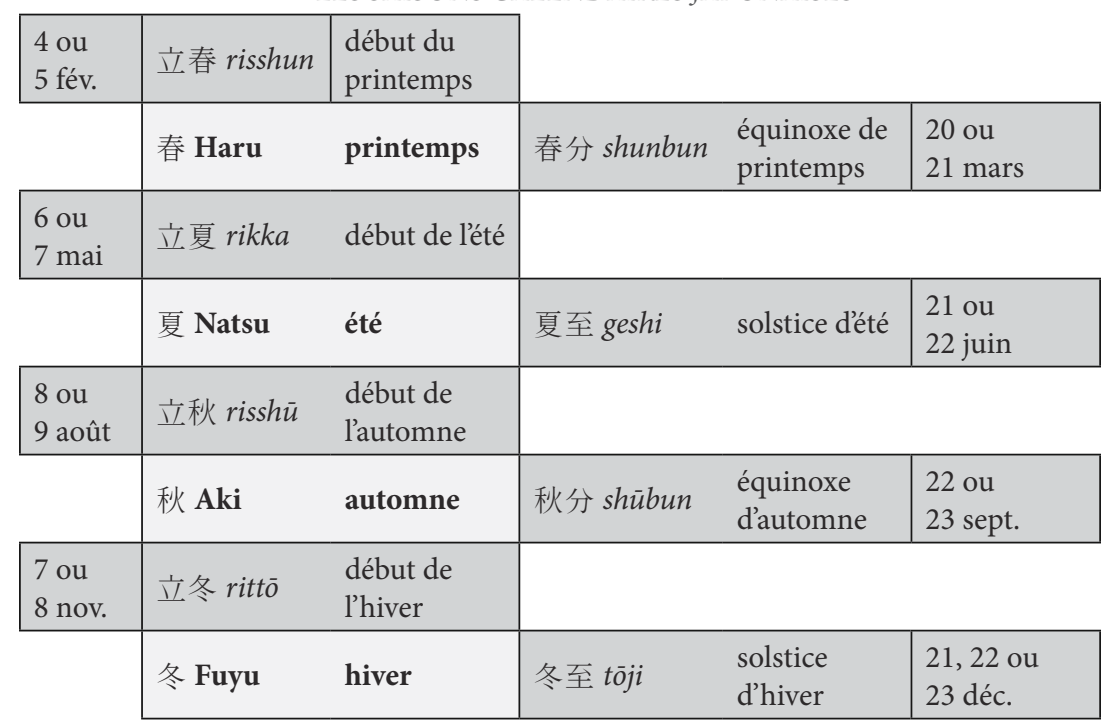




\section{Le cycle sexagésimal}

Le décompte des jours et des années se faisait suivant un cycle sexagésimal correspondant à la combinaison en binômes des dix tiges célestes (jikkan 十干) et des douze branches terrestres (jünishi 十二支) de la cosmologie traditionnelle chinoise. Comme le souligne Marc Kalinowski, l'intégration de ce cycle sexagésimal récurrent inspiré de la cosmogénèse a contribué à construire en Chine une représentation unifiée de l'espace et du temps, et à ancrer la croyance en une corrélation intime entre l'ordre de la nature et celui de la société qui explique que « les cycles calendaires étaient investis d'une autorité semblable à celle d'une loi naturelle $»^{21}$.

Les dix tiges célestes sont obtenues par le dédoublement des cinq « agents » de la cosmologie chinoise (le bois, $k i$ 木; le feu, $h i$ 火 ; la terre, tsuchi 土; le métal ${ }^{22}, k a$ 金; l'eau, mizu 水) en aîné ( $e$ 兄) et cadet (to 弟). Ce couplage aîné-cadet est une transposition de la doctrine taoïste binaire du yin et du yang. Le cycle dénaire ${ }^{23}$ ainsi obtenu fut utilisé pour compter les jours par décades (shun 旬).

\section{DOCUMENT 7.}

\section{LES DIX TIGES CÉLESTES}

$\begin{array}{lll}\text { ki noe e } & \text { 甲 } & \text { aîné du bois } \\ \text { ki no to } & \text { 乙 } & \text { cadet du bois } \\ \text { hinoe } & \text { 丙 } & \text { aîné du feu } \\ \text { hi no to } & \text { 丁 } & \text { cadet du feu } \\ \text { tsuchi no e } & \text { 戊 } & \text { aîné de la terre }\end{array}$

21. Marc KalinowsKI, « La cosmologie traditionnelle en Chine », in La Voie du Tao : un autre chemin de l'être, catalogue d'exposition du Grand Palais, 29 mars-5 juillet 2010, Paris, Réunion des musées nationaux, 2010, p. 90.

22. Les dénominations bois et métal sont des références génériques aux mondes végétal et minéral.

23. Le nombre 10 est parfois mis en rapport avec un mythe chinois selon lequel il y avait jadis dix soleils apparaissant à tour de rôle dans le ciel. 


$\begin{array}{lll}\text { tsuchi no to } & \text { 己 } & \text { cadet de la terre } \\ \text { kano e } & \text { 庚 } & \text { aîné du métal } \\ \text { ka no to } & \text { 辛 } & \text { cadet du métal } \\ \text { mizuno e } & \text { 壬 } & \text { aîné de l'eau } \\ \text { mizu no to } & \text { 癸 } & \text { cadet de l'eau }\end{array}$

Les douze branches terrestres correspondent aux douze animaux de l'astrologie chinoise ${ }^{24}$. Elles furent également utilisées pour compter les heures de la journée et les mois de l'année. Dans un mois de trente jours, le jour associé à une tige céleste revenait donc trois fois et celui associé à un animal deux ou trois fois ${ }^{25}$.

Les jours du mois et les années faisaient ainsi l'objet d'une double numérotation : numéro d'ordre dans la lunaison (ou dans l'ère pour les années) et numéro d'ordre dans le cycle sexagésimal. Le décompte des jours dans le cycle sexagésimal se poursuivait indépendamment des mois ou des années, si bien qu'il n'y avait pas correspondance d'une année sur l'autre entre ces décomptes parallèles.

\section{Élaboration d'un calendrier luni-solaire pour une année donnée (exemple de réalisation pour les années 2016-2017)}

Pour comprendre le fonctionnement pratique du calendrier luni-solaire et resituer certaines célébrations dans leur contexte originel, nous proposons dans cette partie d'expliquer comment mettre en correspondance le calendrier grégorien des années 2016 et 2017 avec le calendrier luni-solaire de Tenpō. Ces deux années présentent l'avantage d'inclure une année « normale » de douze mois et une année particulière de treize mois.

Le calendrier de Tenpō est basé sur trois règles principales :

1) Le $1^{\text {er }}$ jour du mois est le jour de la nouvelle lune (NL).

2) Un mois porte le même numéro que le souffle médian qu'il contient. Le mois contenant l'équinoxe de printemps sera donc toujours le $2^{\mathrm{e}}$ mois, et celui

24. Rat (ne 子), bœuf (ushi 丑), tigre (tora 寅), lièvre ( $u$ 卯), dragon (tatsu 辰), serpent ( $m i$ 巳), cheval (uma 午), bélier (hitsuji 未), singe (saru 申), coq (tori 西), chien (inu 戌) et sanglier $(i$ 亥).

25. Voir Francine Hérail, op. cit., 1986, p. 19 ; ou Marc Kalinowski, op. cit., 2010, p. 90, pour un tableau combinatoire présentant l'ordre du cycle sexagésimal. 
contenant le solstice d'hiver le $11^{\mathrm{e}}$ mois. Lorsqu'une NL et un souffle médian se produisent le même jour, on considère que le nouveau mois contient le souffle même si celui-ci est antérieur à la NL.

3) Un mois qui ne contient pas de souffle médian est appelé mois intercalaire et porte le même numéro que le mois précédent.

Une conséquence importante de ces règles est que le Nouvel An correspond à la seconde nouvelle lune après le solstice $d$ 'hiver.

Dans beaucoup de calendriers prenant en compte le cycle du soleil, le début de l'année est fixé à des moments charnières comme les solstices ou les équinoxes (équinoxe d'automne chez les Phéniciens, solstice d'été à Athènes). Le printemps, symbole du renouveau de la nature, a souvent été choisi par les sociétés agricoles des pays tempérés. Celles-ci ont alors opté soit pour le solstice d'hiver (chez les Gaulois), moment où les jours commencent à rallonger, soit pour l'équinoxe de printemps (en Perse), instant où la durée du jour commence à excéder celle de la nuit. Le calendrier chinois, lui, a fixé une date située à mi-chemin entre le solstice d'hiver et l'équinoxe de printemps.

Dans le premier calendrier romain, l'année commençait en mars. Notre calendrier, qui en est issu, en garde la trace dans les noms des mois de septembre, octobre, novembre et décembre qui indiquaient leur rang dans l'année (respectivement $7^{\mathrm{e}}$, $8^{e}, 9^{e}$ et $10^{e}$ mois de l'année). C'est en 153 av. J.-C. que le premier janvier est devenu le premier jour de l'année. Cette réforme, peu appliquée à l'époque, a été confirmée en 46 av. J.-C. par Jules César mais cette date ne s'imposera définitivement en France qu'en 1564 par un décret de Charles IX. La réforme du pape Grégoire XIII qui, en 1582, a donné naissance au calendrier grégorien universellement utilisé aujourd'hui ne modifiera pas cette date du début de l'année proche du solstice d'hiver.

Pour la réalisation pratique du calendrier de Tenpō pour 2016 et 2017, il faut relever les dates des nouvelles lunes et des souffles médians sur des éphémérides ${ }^{26}$ et mettre les unes en regard des autres (voir document 8).

26. Ces événements astronomiques se produisent aux mêmes instants sur tous les points de la terre. Mais, pour le même instant, l'heure n'est pas la même suivant la longitude du lieu (décalage horaire). En raison de cette universalité, n’importe quelle société astronomique est en mesure d'en fournir les dates. Toutefois, les souffles étant un concept sino-japonais, seuls des sites asiatiques vont nous fournir les informations les concernant. 
On observe d'abord que pour 2016, la lunaison se déployant du 8 février au 9 mars contient le premier souffle médian « eau de pluie » (雨水); il s'agit donc du $1^{\text {er }}$ mois de l'année. On constate ensuite que chaque mois lunaire contient un souffle médian et la numérotation se poursuit ainsi jusqu'au $12^{\mathrm{e}}$ mois qui s'étendra du 29 décembre 2016 au 28 janvier 2017 et qui contient le souffle « grands froids $\gg$ (大寒).

L'année suivante débutera ce 28 janvier. Le 23 juillet 2017 se produit alors la concomitance d'une nouvelle lune et du souffle « grandes chaleurs 》 (大暑). La règle $\mathrm{n}^{\circ} 2$ indique que le souffle appartient au nouveau mois. Par conséquent, le mois lunaire s'étendant du 24 juin au 23 juillet ne contient pas de souffle médian, et d'après la règle $n^{\circ} 3$ il s'agit d'un mois intercalaire (閏月) qui portera le même numéro que le mois précédent, soit le 5 . Cette année comportera donc treize mois. Le Nouvel An suivant aura lieu le 16 février 2018.

Affecter le même numéro que le mois précédent au mois intercalaire permet de garder la correspondance entre les mois et les souffles et donc d'ancrer profondément la composante solaire dans le calendrier lunaire.

D’après les éphémérides du 23 juillet, le souffle « grandes chaleurs » (大 暑) se produit à $0 \mathrm{~h} 15$ et la NL à $18 \mathrm{~h} 45$ (heures légales japonaises). Si l'on tenait compte de l'heure, on devrait alors considérer que c'est le mois débutant au 23 juillet qui ne contient pas de souffle. Dans ce cas, le mois débutant au 24 juin serait le $6^{\mathrm{e}}(6$ 月) et le mois suivant serait le mois intercalaire 6 bis (閏 6 月). 
MESURER LE TEMPS AU JAPON

DOCUMENT 8.

LE CALENDRIER JAPONAIS CORRESPONDANT AUX ANNÉES 2016 ET 2017

\begin{tabular}{|c|c|c|c|c|c|}
\hline \multicolumn{2}{|c|}{2016} & \multicolumn{2}{|c|}{2017} & \multicolumn{2}{|c|}{2018} \\
\hline 中気 & NL & 中気 & $\mathrm{NL}$ & 中気 & NL \\
\hline & 10 janv. & 大寒 20 janv. & 12 月 & & 17 janv. \\
\hline 大寒 21 janv. & 12 月 & & 28 janv. & 大寒 20 janv. & 12 月 \\
\hline & 8 fév. & 雨水 18 fév. & 1 月 & & 16 fév. \\
\hline 雨水 19 fév. & 1 月 & & 26 fév. & 雨水 19 fév. & 1 月 \\
\hline & 9 mars & 春分 20 mars & 2 月 & & \\
\hline 春分 20 mars & 2 月 & & 28 mars & & \\
\hline & 7 avril & 穀雨 20 avril & 3 月 & & \\
\hline 穀雨 20 avril & 3 月 & & 26 avril & & \\
\hline & 7 mai & 小満 $21 \mathrm{mai}$ & 4 月 & & \\
\hline 小満 20 mai & 4 月 & & 26 mai & & \\
\hline & 5 juin & 夏至 21 juin & 5 月 & & \\
\hline 夏至 21 juin & 5 月 & & 24 juin & & \\
\hline & 4 juil. & & 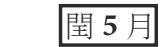 & & \\
\hline 大暑 22 juil. & 6 月 & 大暑 23 juil. & 23 juillet & & \\
\hline & 3 août & & 6 月 & & \\
\hline 処暑 23 août & 7 月 & & 22 août & & \\
\hline & $1^{\text {er }}$ sept. & 処暑 23 août. & 7 月 & & \\
\hline 秋分 22 sept. & 8 月 & & 20 sept. & & \\
\hline & $1^{\mathrm{er}}$ oct. & 秋分 23 sept. & 8 月 & & \\
\hline 霜降 23 oct. & 9 月 & & 20 oct. & & \\
\hline & 31 oct. & 霜降 23 oct. & 9 月 & & \\
\hline 小雪 22 nov. & 10 月 & & 18 nov. & & \\
\hline & 29 nov. & 小雪 22 nov. & 10 月 & & \\
\hline 冬至 21 déc. & 11 月 & & 18 déc. & & \\
\hline & 29 déc. & 冬至 22 déc. & 11 月 & & \\
\hline
\end{tabular}

Ces résultats permettent de compter le nombre de jours de chaque mois et de chacune de ces deux années. 
DOCUMENT 9.

DÉCOMPTE DES JOURS POUR 2016 ET 2017

DANS LE CALENDRIER LUNI-SOLAIRE

2016

\begin{tabular}{|c|c|c|c|c|c|c|c|c|c|c|c|}
\hline 1月 & 2月 & 3月 & 4月 & 5月 & 6月 & 7月 & 8月 & 9月 & 10月 & 11月 & 12月 \\
\hline 30 & 29 & 30 & 29 & 29 & 30 & 29 & 30 & 30 & 29 & 30 & 30 \\
\hline
\end{tabular}

7 mois de 30 jours et 5 mois de 29 jours, soit une année de 355 jours.

2017
\begin{tabular}{|c|c|c|c|c|c|c|c|c|c|c|c|}
\hline 1月 & 2 月 & 3月 & 4 月 & 閏 5月 & 6 月 & 7月 & 8月 & 9月 & 10月 & 11 月 & 12 月 \\
\hline 29 & 30 & 29 & 30 & 29 & 30 & 29 & 30 & 29 & 30 & 30 & 30 \\
\hline
\end{tabular}

7 mois de 30 jours et 6 mois de 29 jours, soit une année de 384 jours.

Comme tous les calendriers, celui-ci réclame parfois quelques ajustements. On a vu dans la quatrième partie que l'intervalle entre deux souffles médians pouvait atteindre un minimum de 29,44 jours et donc être inférieur à la durée d'une lunaison (29,53 jours). Ce phénomène peut donc conduire à ce qu'un mois lunaire contienne deux souffles médians. Mais cet événement s'accompagne de la survenue d'un mois lunaire ne contenant pas de souffle médian. En conséquence, ce dernier ne sera pas considéré comme un mois supplémentaire et viendra compenser le mois contenant deux souffles médians.

\section{Célébration des fêtes traditionnelles dans le calendrier actuel}

Faute d'une bonne compréhension des phénomènes, les discours sur l'ancien calendrier relayent parfois certaines idées reçues qui ne correspondent pas à la réalité. On entend notamment que l'année commençait au risshun. Or, il s'agit d'un événement solaire qui ne coïncide avec une nouvelle lune qu'une fois tous les 19 ans (supra « Partie lunaire »).

Le risshun a lieu le 3 ou le 4 février tandis que le début de l'année oscille entre le 21 janvier et le 20 février. Le début du printemps peut donc être antérieur ou 
postérieur au début de l'année. Bernard Frank fait remarquer l'importance accordée par les poètes à ce « jeu calendérique » :

Lorsque le «point de départ du printemps », qui marquait officiellement l'apparition du renouveau, survenait après le « jour initial », dans la nouvelle année commencée, il semblait apporter à celle-ci, fût-ce tardivement, une note d'accord attendue, mais lorsqu'il survenait avant, tandis que n'était pas encore achevée la « vieille année » (furu-toshi), on éprouvait, tout le temps que durait ce chevauchement, l'impression d'une étrange dualité de règne ${ }^{27}$.

Un raccourci fréquent consiste également à penser que toutes les fêtes calendaires se trouvent désormais « avancées 》 d'environ un mois (durée moyenne correspondant au décalage entre les dates de Nouvel An) et que, pour cette raison, elles seraient désynchronisées avec les phénomènes naturels auxquels elles étaient associées. Cette vision est inexacte car il faut bien distinguer les fêtes liées à des phénomènes lunaires ou à des dates calendaires données (telles que le $3^{\mathrm{e}}$ jour du $3^{\mathrm{e}}$ mois) de celles qui sont liées aux saisons pour lesquelles il y a toujours conjonction avec le phénomène astronomique. C'est en effet la partie lunaire du calendrier qui a été affectée, et la première conséquence est que le décompte des mois et des jours n'est plus synchronisé avec le cycle des lunaisons. Mais, suivant la nature de l'événement, le décalage n'aura pas la même signification et nous aimerions réfléchir ici aux répercussions du changement de calendrier sur la célébration des différentes fêtes traditionnelles qui rythment la vie des Japonais. Quelle est la nature des décalages résultant de ce changement de calendrier et quels sont les aménagements pragmatiques qui ont été opérés ?

Envisageons tout d'abord le cas des fêtes dont le jour de célébration est associé à une date dont les chiffres portent une symbolique numérologique forte comme le jour de l'An ou les sekku. Ces derniers sont des jours particuliers correspondant, à l'origine, à divers rites divinatoires ou religieux (purification, etc.). Dans le processus de transmission de la Chine au Japon, puis à l'intérieur du Japon lui-même, ces célébrations ont subi au fil des siècles de nombreuses transformations pour devenir

27. Bernard FrANK, op. cit., 2011, p. 304. 
des fêtes populaires païennes. Elles étaient à l'origine au nombre de cinq, mais les Japonais ne célèbrent plus guère aujourd'hui que Jōshi no sekku 上巳の節句, Fête du $1^{\text {er }}$ jour du serpent, plus connue sous le nom de momo no sekku 桃の節句, Fête des pêchers ( $3^{\mathrm{e}}$ jour du $3^{\mathrm{e}}$ mois), tango no sekku 端午の節句, Fête du $1^{\text {er }}$ jour $\mathrm{du}$ bœuf ( $5^{\mathrm{e}}$ jour du $5^{\mathrm{e}}$ mois) et tanabata $七$ 夕, Fête de la $7^{\mathrm{e}}$ nuit ( $7^{\mathrm{e}}$ nuit du $7^{\mathrm{e}}$ mois). Pour celles-ci, le respect de la symbolique numérologique a semblé nécessaire et elles ont été (dé)placées de manière purement comptable dans le nouveau calendrier. Elles « arrivent » donc avec anticipation par rapport à l'ancien calendrier et le décalage qui en résulte est d'ordre saisonnier. En d'autres termes, lorsqu'elles se produisent, il est trop tôt pour pouvoir observer les phénomènes naturels auxquels sont traditionnellement associées ces fêtes (par exemple la floraison du pêcher pour momo no sekku ou celle de l'iris pour tango no sekku). Mais cela ne revêt pas une trop grande importance. Comme on peut le vérifier chaque année avec la progression du « front des cerisiers », ces phénomènes naturels ne se produisent pas au même moment dans tout le pays et la coïncidence était donc déjà approximative dans l'ancien calendrier. D'ailleurs ces fêtes, importées de Chine, avaient depuis longtemps perdu leurs fondements astronomiques au profit de pratiques culturelles symboliques.

La fête de $o$-bon お盆, Fête des Morts, fixée au $15^{\mathrm{e}}$ jour du $7^{\mathrm{e}}$ mois dans l'ancien calendrier (ainsi que, dans certaines régions, tanabata avec laquelle elle est en relation) a été placée avec un mois de décalage, soit aux environs du 15 août dans le nouveau calendrier, suivant la pratique dite du tsuki okure (月遅れ, report d'un mois). Le 15 juillet correspondant à la saison des pluies, on peut penser que le souci de conserver l'environnement climatique estival caractéristique des célébrations de $o$-bon a présidé à ce choix. Certains Japonais soucieux de perpétuer la tradition continuent de célébrer $o$-bon à la mi-juillet mais, stricto sensu, le respect de la tradition serait plutôt dans cette pratique du tsuki okure.

Certaines célébrations étaient également fixées à des dates calendaires précises, non pas pour la symbolique de la date elle-même, mais en raison du phénomène astronomique auquel elles étaient associées. La $15^{\mathrm{C}}$ lune (jügoya) observée dans la nuit du $15^{\mathrm{e}}$ au $16^{\mathrm{e}}$ jour du $8^{\mathrm{e}}$ mois ou la $13^{\mathrm{e}}$ lune (jüsan.ya) fixée à la nuit du $13^{\mathrm{e}}$ au $14^{\mathrm{e}}$ jour du $9^{\mathrm{e}}$ mois en sont quelques exemples. Pour pouvoir observer le phénomène en question, il a donc fallu transposer ces dates dans le calendrier grégorien. Il en résulte un certain décalage entre le nom de la fête et le concept qu'elle 
désigne, ce qui donne dorénavant des allures de nom propre aux termes de jūsan. ya et jügoya. C'est une preuve de la survivance du calendrier luni-solaire au Japon, non pas comme calendrier officiel de computation du temps, mais plutôt comme une référence seconde dans les almanachs.

Une autre catégorie de célébrations est liée aux autres souffles (zassetsu 雑節) tels que setsubun 節分, higan 彼岸, hachijūhachi-ya 八十八夜, doyō 土用, etc. Ces dates fournissent des repères saisonniers venant s'ajouter aux 24 souffles. Elles relèvent donc de la composante solaire du calendrier et, à ce titre, il y a toujours corrélation entre ces phénomènes astronomiques (et leurs incidences climatiques) et leur date. Ce qui a changé dans le nouveau calendrier, c'est qu'au lieu d'être « mobiles », ces événements se produisent dorénavant à dates fixes.

Higan désigne les deux périodes de 7 jours encadrant les équinoxes de printemps et d'automne. Celles-ci sont marquées par des cérémonies d'hommage aux défunts issues de certains rites de la secte bouddhique de la Terre pure (jödoshī 浄土宗) dont le paradis se situe à l'ouest, dans la direction précise où se couche le soleil lors des équinoxes ${ }^{28}$. Aujourd'hui encore, ces périodes sont l'occasion de visites aux cimetières. Elles sont également présentes dans la culture populaire par le dicton : «le froid ou la chaleur ne dure que jusqu'à higan » (atsusa samusa mo higan made 暑さ寒さも彼岸まで) ou par des pâtisseries saisonnières (bota moch $i$ 牡丹餅 au printemps et $o$-hagi 御萩 à l'automne ${ }^{29}$ ).

Doyō désigne la période de 18 jours précédant chaque ritsu. Il y en a ainsi quatre par an, même si ce terme renvoie plus particulièrement au ritsu qui précède le pas-

28. La direction dans laquelle le soleil se couche varie tout au long de l'année. Il se couche précisément à l'ouest lors des deux équinoxes. Après l'équinoxe de printemps, la position s'écarte vers le nord pour atteindre un écart maximum par rapport à l'ouest au solstice d'été. Puis, le point de coucher du Soleil revient à l'ouest lors de l'équinoxe d'automne et dérive ensuite vers le sud avec un écart maximum au solstice d'hiver. L'amplitude des écarts dépend de la latitude du lieu.

29. Ces deux noms désignent une même pâtisserie populaire constituée de mochi fait de riz blanc entouré d'une couche de haricots sucrés de couleur sombre. Alors que dans la pâtisserie japonaise, le haricot rouge est en général au cœur des gâteaux, dans le bota mochi ou le $o$-hagi, la place de ces deux ingrédients est donc en quelque sorte intervertie, évoquant ainsi la symbolique de l'inversion entre la durée du jour et de la nuit au moment de l'équinoxe. 
412 Cahiers d'études japonaises n² 21

sage à l'automne (du 19 juillet au 7 août) et qui correspond au pic annuel de chaleur. Le premier jour est nommé « entrée dans doyō»(doyō no iri 土用の入り) et le dernier jour correspond donc à un setsubun (jointure entre deux saisons). Durant cette période de 18 jours, le jour du bœuf (ushi no hi \#丑の日) revient à une ou deux reprises (ce sera le 30 juillet en 2016). Depuis l'époque d'Edo, une coutume populaire dont l'initiative est attribuée à Hiraga Gennai 平賀源内 veut que l'on déguste ce jour-là de l'anguille ${ }^{30}$.

Comme le rappelle Francine Hérail, les fêtes des sanctuaires étaient en général fixées au $1^{\text {er }}$ ou au $2^{\mathrm{e}}$ jour du mois correspondant à l'un des douze animaux du calendrier ${ }^{31}$. Cette tradition s'est perpétuée et subsiste localement dans des fêtes populaires dont les dates sont mobiles suivant les années. À Tōkyō, le marché du $\operatorname{coq}$ (tori no ichi 酉の市) qui se déroule au sanctuaire d'Asakusa le jour du coq du $11^{\mathrm{e}}$ mois est un exemple de tradition qui attire toujours une foule immense.

Pour des raisons diverses, certains aménagements pragmatiques ont ainsi été opérés lors du changement de calendrier et il n'est pas toujours facile de « débrouiller l'écheveau » des fêtes populaires du calendrier japonais.

\section{Conclusion}

Mis à part le calendrier musulman purement lunaire et le calendrier julien purement solaire, presque tous les calendriers ont cherché à concilier l'inconciliable, à savoir harmoniser les cycles lunaires et solaires. Tous sont parvenus à un compromis au moyen de jours ou de mois intercalaires basés sur des cycles plus ou moins précis. Le choix des intercalations, qui comporte une part d'arbitraire, a souvent donné lieu à des luttes de pouvoir, ce qui a fréquemment conduit à des

30. Pour lutter contre une baisse des affaires en été, Hiraga Gennai 平賀源内 aurait conseillé à un marchand d'anguilles d'accrocher une affiche précisant : « Aujourd'hui, c'est le jour du bœuf ». La légende veut que cette opération de «marketing » ait rencontré un vif succès. Cela s'explique par les qualités nutritives de l'anguille et le fait que la coutume veuille que l'on mange traditionnellement un aliment commençant par la syllabe « u » le jour du bœuf (ushi no hi). Parmi les autres aliments prisés à cette date, on peut citer le bœuf (ushi), le cheval (uma), le udon, le melon (uri), etc.

31. Francine Hérail, op. cit., 1986, p. 18. 
bouleversements importants. Dans le calendrier chinois et son pendant japonais, la méthode d'intercalation simple et cependant très rigoureuse basée sur la présence ou l'absence d'un souffle dans une lunaison est peut-être un des facteurs ayant permis d'éviter les changements brutaux au profit d'ajustements fins basés sur l'amélioration des méthodes d'observation astronomique. L'ensemble de rites, cérémonies, interdictions et obligations sévères prenant appui sur le calendrier et très strictement respecté a aussi probablement contribué à son maintien sur une très longue période ${ }^{32}$.

De par la croyance en une corrélation entre l'ordre naturel et celui de la société, le calendrier luni-solaire a ainsi servi de ciment à la culture japonaise. Aujourd'hui encore les Japonais restent très attachés aux calendriers qui constituent toujours un produit de consommation très prisé et, près de cent cinquante ans après son abandon, le calendrier luni-solaire est toujours vivant dans la société japonaise comme une référence calendaire seconde servant à déterminer les dates de fêtes traditionnelles ou de célébrations privées (mariage, commémoration, etc.).

\section{Bibliographie}

BIÉmont Emile, Rythmes du temps : astronomie et calendriers, Bruxelles, De Boeck, 2000.

Caillet Laurence, Fêtes et rites des quatre saisons au Japon, Cergy, Publications Orientalistes de France, 2002.

Frank Bernard, Kata-imi et kata-tagae : étude sur les interdits de direction à l'époque Heian, Paris, Collège de France, 1998.

32. Le calendrier hébreu également luni-solaire et avec des contraintes très importantes connaît lui aussi une longévité exceptionnelle mais il n'est utilisé que dans le cadre de la religion. 
FRANK Bernard, Démons et jardins : aspects de la civilisation du Japon ancien, Paris, Collège de France - Institut des Hautes Études Japonaises, 2011.

Hérail Francine, Histoire du Japon : des origines à la fin de Meiji, Paris, Publications Orientalistes de France, 1986.

Kalinowski Marc, «La cosmologie traditionnelle en Chine », in La Voie du Tao : un autre chemin de l'être, catalogue d'exposition du Grand Palais, 29 mars-5 juillet 2010, Paris, Réunion des musées nationaux, 2010, p. 85-95.

YAmAZAKi-Dubois Sylvie, BazAntay Jean, « Le calendrier luni-solaire japonais », Les Cabiers Clairaut, nº 146, Nice, CLEA, 2014a, p. 2-6.

Yamazaki-Dubois Sylvie, Bazantay Jean, « Le calendrier luni-solaire japonais », Les Cabiers Clairaut, $\mathrm{n}^{\circ}$ 147, Nice, CLEA, 2014b, p. 30-35.

\section{Dictionnaire et encyclopédie}

OKada Yoshirō 岡田芳朗, Akune Suetada 阿久根末忠 (dir.), Gendai koyomi yomitoki jiten 現代暦読み解き辞典 (Dictionnaire compréhensif des calendriers contemporains), Tōkyō, Kashiwa shobō 柏書房, 1993.

Koyomi No KAI (dir.), Koyomi no hyakka jiten 暦の百科事典 (Encyclopédie des calendriers), Tōkyō, Shinjinbutsuōrai-sha 新人物往来社, 1986.

\section{Sites Internet}

Kokuritsu Tenmondai 国立天文台 (National Astronomical Observatory of Japan), http://www.nao.ac.jp/ [octobre 2014].

Institut de mécanique céleste et de calcul des éphémérides (IMCCE) Connaissance du temps, Ephémérides générales http://www.imcce.fr/ [octobre 2014]. 
MESURER LE TEMPS AU JAPON

Tentai, tenmon, koyomi, chizu, kishō arakaruto 天体 天文 暦 地図 気象 アラカルト (Corps célestes, astronomie, calendrier, cartes et climats à la carte) http://homepage2.nifty.com/yamakatsu/index_astro.html [octobre 2014].

Résumé : Parmi les réformes emblématiques de la politique d'occidentalisation conduite par le gouvernement de Meiji, figure l'adoption du calendrier grégorien en 1873. Jusqu'alors la computation du temps s'effectuait suivant des calendriers luni-solaires d'inspiration chinoise dont le dernier en vigueur était celui de Tenpō. Toutefois, près de 150 ans après son abandon, les références à l'ancien calendrier sont omniprésentes dans la société japonaise et la connaissance du fonctionnement du luni-solaire reste une clef d'accès à une bonne compréhension de la culture japonaise. Cet article offre quelques repères en expliquant le fonctionnement de l'ancien calendrier, et notamment sa composante solaire avec le système des 24 souffles et l'introduction d'un mois intercalaire permettant d'éviter le glissement des saisons, défaut propre à un calendrier purement lunaire. À titre d'illustration, le fonctionnement pratique du calendrier luni-solaire est expliqué par la mise en correspondance du calendrier grégorien des années 2016 et 2017 avec le calendrier luni-solaire de Tenpō. La mise en parallèle des deux systèmes permettra d'éclairer l'anachronie de certaines pratiques culturelles actuelles.

Mots-clés : calendrier, calendrier luni-solaire/grégorien, saisons, souffles, fêtes et rites, calendrier de Tenpō

Abstract: The adoption of the Gregorian calendar in 1873 was one of the Meiji government's emblematic policies of Westernization. Until then, time computation had been based on lunisolar calendars of Chinese origin; the last calendar to be used was known as the "Tenpo calendar". However, nearly 150 years after it was abandoned, references to the old calendar (kyūreki) are everywhere in Japanese society and correct knowledge of how it functioned remains a key to accurately understanding Japanese culture. This article will provide clear explanations of the old calendar, in particular its solar component with the system of twenty-four solar divisions (sekki), and the introduction of an intercalary month to prevent seasonal shifts - a major weakness of purely lunar calendars. As an illustration, we will explain how to map 


\section{CIPANGO}

416 Cahiers d'études japonaises nº 21

the Gregorian calendar for 2016 and 2017 using the lunisolar Tenpo calendar era. A comparison of the two systems will highlight the anachronistic nature of certain current cultural practices.

Keywords: Calendar, Lunisolar/Gregorian Calendar, Seasons, sekki, Festivals and Rites, Calendar of Tenpō

キーワード：暦, 太陰太陽歴, グレゴリオ暦, 季節, 節気, 年中行事, 天 保歴 